\title{
Laparoscopic Surgery Combined with GnRH Agonist in Endometriosis
}

\author{
Yu Yang1, Weidong Zhu1, Shuqiu Chen1, Guangyuan Zhang1, Ming Chen'1 and Yuhong Zhuang2
}

\begin{abstract}
Objective: To determine the efficacy of laparoscopic surgery combined with $\mathrm{GnRH}$ agonist ( $\mathrm{GnRH}-\mathrm{a})$ in treating infertile patients with endometriosis (EMs), and analyze its effect on the levels of serum hormones and inflammatory factors of the patients.

Study Design: An experimental study.

Place and Duration of Study: The Department of Urology, Zhongda Hospital, Southeast University, Jiang Su, China, from January 2015 to March 2016.

Methodology: One hundred and thirty infertile patients with EMs were randomly divided into a control group and an observation group with 65 cases in each group. Patients in the control group were treated by laparoscopic surgery, while patients in the observation group were added by the subcutaneous injection of $\mathrm{GnRH}-\mathrm{a}$, based on the treatment of the control group. Therapeutic effects, recurrence rates, and pregnancy rates between two groups were compared. Moreover, the levels of serum hormones estradiol $\left(E_{2}\right)$, luteinizing hormone $(\mathrm{LH})$, follicle stimulating hormone (FSH), inflammatory factors interleukin 17 (IL-17), interleukin 6 (IL-6), and tumor necrosis factor- $\alpha$ (TNF- $\alpha$ ) between two groups after treatment were compared.

Results: The total effective rate and pregnancy rate in the observation group were higher than those of the control group $(p=0.009$ and 0.002 , respectively), and the recurrence rate was lower than that of the control group $(p=0.008)$. After treatment, levels of serum $\mathrm{E}_{2}, \mathrm{LH}, \mathrm{FSH}$ in the observation group were lower than those of the control group (all $p<0.001$ ). Levels of serum IL-17, IL-6 and TNF- $\alpha$ were lower than those of the control group (all $p<0.001$ ). There was no statistically significant difference in adverse reactions between the two groups $(p=0.730)$.

Conclusion: Laparoscopic surgery combined with $\mathrm{GnRH}-\mathrm{a}$ is a more effective treatment modality in endometriosis. It can reduce the serum levels of inflammatory factors in patients, improve their hormone level and pregnancy rate, and reduce the recurrence rate.
\end{abstract}

Key Words: Laparoscopy, GnRH agonist, Endometriosis, Infertility, Hormones, Inflammatory factors.

\section{INTRODUCTION}

Endometriosis (EMs) is a clinically common gynaecological disorder that can grow or develop under the influence of sex hormones and is a condition in which the glands or interstitium of the endometrium develop outside the endometrium. ${ }^{1}$ Most ectopic endometrium appears in the peritoneal surface of adjacent organs, but also can grow in areas away from the uterus, such as the groin, diaphragm. The clinical manifestations of EMs are dysmenorrhea, pelvic pain, sexual intercourse pain and menstrual disorders. According to the location of the disease, intestinal, urinary tract symptoms, and even acute abdomen may occur. 2,3 EMs is closely related to female infertility. ${ }^{4}$ Relevant documents show that there are many factors contributing to EMs combined with infertility, which may be related to fallopian tubes, ovaries, endometrium, ovulation and hormonal secretion. ${ }^{5}$

Department of Urologyl / Gynaecology², Zhongda Hospital, Southeast University, Jiang Su, China

Correspondence: Dr. Ming Chen, Department of Urology, Zhongda Hospital, Southeast University, Jiang Su, 210000, China

E-mail:jlc1r1@163.com

Received: April 26, 2018; Accepted: October 25, 2018
In recent years, laparoscopic technology has been widely used in clinical practice. In infertile patients with endometriosis, laparoscopic surgery can completely remove the lesion tissue. The operation time is relatively short, which is beneficial to the recovery of patients. However, it is difficult to completely clear the tissue of serious infiltration or small retroperitoneal lesions by laparoscopic surgery, so it is prone to postoperative recurrence.6,7 Therefore, laparoscopic surgery is the main treatment in clinic, and always combined with adjuvant medical therapy. 8 Gonadotropin-releasing hormone agonist $(\mathrm{GnRH}-\mathrm{a})$ plays an important role in the treatment of endometriosis by reducing estrogen levels to promote the progressive atrophy of ectopic endometrium. ${ }^{9}$

The purpose of this study was to investigate the effects of laparoscopic surgery combined with $\mathrm{GnRH}-\mathrm{a}$ on serum hormones and inflammatory factors in infertile patients with EMs.

\section{METHODOLOGY}

This study was conducted at the Department of Urology, Zhongda Hospital, Southeast University, Jiang Su, China, from January 2015 to March 2016, after approval 
from the Hospital Ethical and Research Committee. A total of 130 infertile patients with EMs were selected as the research objects. Inclusion criteria were the diagnosis of endometriosis confirmed by laparoscopy or B-ultrasound examination, postoperative biopsy, normal sexual life, and without contraception; but no pregnancy for more than one year, and having indications for laparoscopic surgery. Exclusion criteria were other causes of dysmenorrhea, abnormal menstruation and other symptoms, other diseases such as pelvic floor dysfunction, severe cardiovascular, hepatic or renal dysfunction, recent use of hormones, allergy to the medicines used in this study, and patient's spouse suffering from sexual dysfunction or seminal abnormality. The 130 patients were randomly divided into the control group and the observation group, 65 cases in each group.

Patients in the control group were treated with laparoscopic surgery at 3-7 days after the end of menstruation under general anesthesia with standard procedure. Laparoscopic surgery was performed using the threehole method. Under the microscope, the cysts were removed, and ectopic lesions were peeled off. Debridement was performed for pelvic adhesions. Small lesions were subjected to electrocautery destruction measures as far as possible to avoid recurrence due to residual conditions. For ovarian endometriosis, it was necessary to thoroughly remove ovarian cysts; chocolate cysts were perforated on the surface of the ovary, liquid removed and the cyst peeled. Methylene blue test was performed as necessary. In the observation group, the patients were injected sub-cutaneously with $3.75 \mathrm{mg}$ of gonadotropin-releasing hormone agonist (triptorelin acetate for injection) for six months after laparoscopic surgery.

After the treatment had ended, treatment results of the two groups of patients were observed. Excellent result was when hysterosalpingography showed unilateral or bilateral patency, ultrasound showed that all pelvic masses disappeared, and other clinical symptoms disappeared completely. Results were labelled valid when hystero-salpingography showed unilateral patency, and ultrasound showed that all pelvic masses became smaller and the clinical symptoms were relieved. Invalid results of hysterosalpingography showed that both tubes were blocked, and there was no change in pelvic mass. In addition, clinical symptoms did not ease or even aggravate. After treatment, levels of serum estradiol $\left(E_{2}\right)$, luteinizing hormone (LH), follicle stimulating hormone (FSH) and other hormone indexes of patients were detected by automatic microparticle radio-immunoassay system. Serum levels of inflammatory factor interleukin-17 (IL-17), interleukin-6 (IL-6) and tumor necrosis factor- $\alpha$ (TNF- $\alpha$ ) were determined by ELISA. Adverse reactions such as nausea, vomiting, rash and injection pain were recorded in the two groups. All the patients in the two groups were followed up for two years. The recurrence rate and pregnancy rate of two groups were compared.

Data was entered and analysed by using SPSS version 21.0. Measurement data was indicated in $x \pm s$. Independent-sample t-test was used to compare the outcomes between two groups. Count data was expressed by $n(\%)$, and $X^{2}$ test was used to compare count data. P-value of less than 0.05 was considered significant.

\section{RESULTS}

The inducted 130 patients were 24-35 years old, with an average age of $28.73 \pm 1.92$ years. The course of disease ranged from 3 months to 3 years, with an average of $1.78 \pm 0.46$ years. The diameter of the cyst ranged from 3-10 cm, with an average of $5.65 \pm 1.31 \mathrm{~cm}$. According to endometriosis staging, 80 (61.54\%) cases had stage III and $50(38.46 \%)$ cases had stage IV disease.

The total effective rate of the observation group was $96.92 \%$ (63 cases), which was higher than that of the control group $(83.08 \%, 54$ cases, $p=0.009$, Table I).

After treatment, the levels of $E_{2}, \mathrm{LH}$, and $\mathrm{FSH}$ in the observation group were lower than those of the control group (all $p<0.001$, Table II); serum levels of IL-17, IL-6 and TNF- $\alpha$ in the observation group were lower than those of the control group (all $p<0.001$, Table III).

The pregnancy rate in the observation group was $30.77 \%$ (20 cases), significantly higher than that of the control group $(9.23 \%$, six cases, $p=0.002)$. The recurrence rate was $1.54 \%$ ( 1 case), significantly lower than that of the control group $(13.85 \%, 9$ cases, $p=0.008)$.

Table I: Comparison of clinical effect between the two groups.

\begin{tabular}{|c|c|c|c|c|c|c|}
\hline Groups & $\mathrm{n}$ & $\begin{array}{c}\text { Excellent } \\
\text { [n (\%)] }\end{array}$ & $\begin{array}{c}\text { Valid } \\
\text { [n (\%)] }\end{array}$ & $\begin{array}{l}\text { Invalid } \\
\text { [n (\%)] }\end{array}$ & $\begin{array}{c}\text { Total effective } \\
{[\mathrm{n}(\%)]}\end{array}$ & $p$-value \\
\hline Control group & 65 & $19(29.23)$ & 35 (53.85) & 11 (16.92) & 54 (83.08) & 0.009 \\
\hline Observation group & 65 & $32(49.23)$ & 31 (47.69) & $2(3.08)$ & $63(96.92)$ & \\
\hline
\end{tabular}

Table II: Comparison of level of serum hormones between the two groups.

\begin{tabular}{|c|c|c|c|c|c|c|c|}
\hline \multirow[t]{2}{*}{ Groups } & \multirow[t]{2}{*}{$n$} & \multicolumn{2}{|c|}{ E2 (ng/L) } & \multicolumn{2}{|c|}{$\mathrm{LH}(\mathrm{U} / \mathrm{L})$} & \multicolumn{2}{|c|}{$\mathrm{FSH}(\mathrm{U} / \mathrm{L})$} \\
\hline & & Mean \pm SD & $p$-value & Mean $\pm S D$ & p-value & Mean $\pm S D$ & $p$-value \\
\hline Control group & 65 & $176.45 \pm 3.35$ & $<0.001$ & $4.77 \pm 0.37$ & $<0.001$ & $5.31 \pm 0.51$ & $<0.001$ \\
\hline Observation group & 65 & $94.38 \pm 2.24$ & & $2.05 \pm 0.29$ & & $2.16 \pm 0.43$ & \\
\hline
\end{tabular}


Table III: Comparison of level of serum inflammatory factors between the two groups.

\begin{tabular}{|c|c|c|c|c|c|c|c|}
\hline \multirow[t]{2}{*}{ Groups } & \multirow[t]{2}{*}{$\mathrm{n}$} & \multicolumn{2}{|c|}{ IL-17 (pg/mL) } & \multicolumn{2}{|c|}{ IL-6 (pg/mL) } & \multicolumn{2}{|c|}{ TNF- $\alpha(\mathrm{pg} / \mathrm{mL})$} \\
\hline & & Mean \pm SD & $\mathrm{p}$-value & Mean \pm SD & $\mathrm{p}$-value & Mean $\pm S D$ & $\mathrm{p}$-value \\
\hline Control group & 65 & $15.84 \pm 0.87$ & $<0.001$ & $46.29 \pm 3.35$ & $<0.001$ & $147.31 \pm 15.43$ & $<0.001$ \\
\hline Observation group & 65 & $13.63 \pm 0.55$ & & $42.94 \pm 2.67$ & & $138.46 \pm 7.12$ & \\
\hline
\end{tabular}

The frequency of adverse reactions in the observation group was $6.15 \%$ (4 cases), including three cases of nausea and one case of pain at the injection site. The frequency of adverse reactions in the control group was $7.69 \%$ (5 cases), including two cases of nausea and vomiting each, and one case of vertigo. There was no significant difference in adverse reactions between the two groups $(p=0.730)$.

\section{DISCUSSION}

Endometriosis is an estrogen-dependent disease with an incidence of about $10 \%$ to $15 \%$. It is most common in women of childbearing age, and more than $40 \%$ of patients may be complicated with infertility. ${ }^{10,11}$ In the treatment of endometriosis combined with infertility, laparoscopic surgery has gradually replaced the traditional open surgery because of its advantages of small incision, less trauma, less pain, fewer complications, and good curative effect. At present, laparoscopic surgery has become the first choice for endometriosis. ${ }^{12,13}$ In laparoscopic surgery, the operation field can be clearly distinguished by the magnifying effect of laparoscope, which is convenient to observe the pelvic micro-lesion. Moreover, the oviduct patency can be directly observed by methylene blue solution during the operation. Repeated irrigation of the abdominal cavity after operation can also promote the improvement of pelvic micro-environment.14,15 According to relevant documents, laparoscopic conservative surgery can relieve dysmenorrhea, lower abdominal pain, dyspareunia, menstrual abnormalities, and other symptoms in patients with endometriosis, and eliminate potential malignant tissue.16,17 However, laparoscopic surgery alone cannot effectively remove atypical lesions or deeper lesions, the recurrence rate is higher. Therefore, it is suggested that drug therapy should be combined with laparoscopic surgery in order to promote the atrophy and necrosis of residual lesions and reduce recurrence. ${ }^{18}$

$\mathrm{GnRH}-\mathrm{a}$ has been shown to improve the efficacy of laparoscopic surgery in infertile patients with endometriosis, but the mechanism of action is not fully understood.19,20 The use of triptorelin acetate for injection in this study is a novel long-acting $\mathrm{GnRH}-\mathrm{a}$, with a long half-life and good stability, which play a longterm inhibitory effect on pituitary gonadotropin secretion, and block the hypothalamus-pituitary-ovarian axis (HPOA), promote ovarian hormone levels significantly decreased, induce temporary amenorrhea. Moreover, ectopic lesions without hormone support may atrophy and degenerate.21,22 The data showed that the total effective rate and pregnancy rate in the observation group were higher than the control group, and the recurrence rate was lower than the control group. The $\mathrm{E}_{2}$, LH, and FSH levels in the observation group were significantly better than the control group. It suggests that laparoscopy combined with gonadotropin-releasing hormone agonist is effective in the treatment of endometriosis combined with infertility. After laparoscopic surgery, $\mathrm{GnRH}$-a treatment is able to make the ectopic endometrium without the support of estrogen and inhibit its growth, thereby improving the pelvic environment and reducing the recurrence rate. Moreover, withdrawal can prompt the ovulation function to recover quickly and improve the pregnancy rate, so the pregnancy rate of patients after operation is obviously improved. This study also found that there was no significant difference in adverse reactions between the two groups, suggesting that $\mathrm{GnRH}-\mathrm{a}$ treatment would not increase adverse reactions, and was safe and reliable.

Recent studies have shown that immune factors play an important role in the pathogenesis of endometriosis complicated with infertility; especially, the relationship between cytokines and EMs infertility has become a research hotspot. 23 The level of IL-6 in peripheral blood of EMs patients was significantly higher than normal people, and positively correlated with the degree of disease. 24 TNF- $\alpha$ is a kind of cytokine with a variety of biological activities. Abnormal levels of TNF- $\alpha$ can directly lead to abortion, premature birth, infertility and other diseases. In circulating blood, the ratio of helper T cells to suppressor T cells increased; and IL-17, IL-6 and TNF- $\alpha$, secreted by Th1 and Th2, also increased.

Lymphocytes in peripheral blood of healthy people can recognize endometrial cells, antigens and have reactive hyperplasia. This reaction is reduced in patients with EMs, which may be due to decreased ability to identify lymphocytes. Similarly, cytotoxicity of NK-mediated lymphocytes in peripheral blood to autologous endometrium is reduced. The number of monocytes in peripheral blood did not change, but the activity increased, stimulating the growth of endometrial cells. Elevated IL-17, IL-6, TNF- $\alpha$ and other cytokines in the peripheral blood may promote the development of EMs. The levels of nitric oxide synthase 2 (NOS2) and nitric oxide (NO) in the peritoneal fluid of EMS patients were significantly increased. High level of NOS2 could promote the secretion of a large amount of NO. High levels of NO can influence the function of ovarian ducts and sperm, resulting in infertility. 25 However, IL-17 can increase the production of NOS2 and NO. Therefore, high levels of IL-17 in the blood of EMs patients with infertility may be involved in the infertility process of 
patients with EMs by promoting the production of NOS2 and NO. The results showed that the levels of serum inflammatory factors IL-17, IL- 6 and TNF- $\alpha$ in the observation group were lower than the control group after treatment. It is suggested that $\mathrm{GnRH}$-a therapy after laparoscopic surgery can effectively reduce the levels of serum inflammatory factors IL-17, IL-6 and TNF- $\alpha$, and further improve the condition of patients.

\section{CONCLUSION}

Laparoscopic surgery combined with $\mathrm{GnRH}$-a is a more effective treatment modality in endometriosis, and it can reduce the serum levels of inflammatory factors in patients, improve their hormone level and pregnancy rate, and reduce the recurrence rate.

Acknowledgement: This study was supported by Medical Science and Technology Development Project of Nanjing City in China (YKK16282).

\section{REFERENCES}

1. Salati SA, Raza AA. Endometriosis: A rare cause of appendicitis. J Coll Physicians Surg Pak 2011; 21:304-5.

2. Vercellini $P$, Somigliana E, Viganò $P$, Abbiati A, Barbara $G$, Crosignani PG. Endometriosis: Current therapies and new pharmacological developments.Drugs 2009; 69:649-75.

3. Chen LC, Hsu JW, Huang KL, Bai YM, Su TP, Li CT, et al. Risk of developing major depression and anxiety disorders among women with endometriosis: A longitudinal follow-up study. J Affect Disord 2016; 190:282-5.

4. Ricci $E$, Viganò $P$, Cipriani $S$, Chiaffarino $F$, Bianchi $S$, Rebonato G, et al. Physical activity and endometriosis risk in women with infertility or pain: Systematic review and metaanalysis. Medicine (Baltimore) 2016; 95:e4957.

5. Prescott J, Farland LV, Tobias DK, Gaskins AJ, Spiegelman D, Chavarro JE, et al. A prospective cohort study of endometriosis and subsequent risk of infertility. Hum Reprod 2016; 31:1475-82.

6. Centini G, Afors K, Murtada R, Argay IM, Lazzeri L, Akladios CY, et al. Impact of laparoscopic surgical management of deep endometriosis on pregnancy rate. J Minim Invasive Gynecol 2016; 23:113-9.

7. Soriano D, Bouaziz J, Elizur S, Zolti M, Orvieto R, Seidman D, et al. Reproductive outcome is favorable after laparoscopic resection of bladder endometriosis. J Minim Invasive Gynecol 2016; 23:781-6.

8. Boujenah J, Cedrin-Durnerin I, Herbemont C, Sifer C, Poncelet C. Non-ART pregnancy predictive factors in infertile patients with peritoneal superficial endometriosis. Eur J Obstet Gynecol Reprod Biol 2017; 211:182-7.

9. Boujenah J, Cedrindurnerin I, Herbemont C, Bricou A, Sifer C, Poncelet $C$. Use of the endometriosis fertility index in daily practice: A prospective evaluation. Eur J Obstet Gynecol Reprod Biol 2017; 219:28-34.

10. Donnez J, Binda MM, Donnez O, Dolmans MM. Oxidative stress in the pelvic cavity and its role in the pathogenesis of endometriosis. Fertil Steril 2016; 106:1011-7.

11. Kondo W, Daraï E, Yazbeck C, Panel P, Tamburro S, Dubuisson $\mathrm{J}$, et al. Do patients manage to achieve pregnancy after a major complication of deeply infiltrating endometriosis resection? Eur J Obstet Gynecol Reprod Biol 2011; 154:196-9.

12. Franck C, Poulsen MH, Karampas G, Giraldi A, Rudnicki M. Questionnaire-based evaluation of sexual life after laparoscopic surgery for endometriosis: A systematic review of prospective studies. Acta Obstet Gynecol Scand 2018; 97:1091-104.

13. De Neef A, Cadière GB, Bourgeois P, Barbieux R, Dapri G, Fastrez $M$. Fluorescence of deep infiltrating endometriosis during laparoscopic surgery: A preliminary report on 6 cases. Surg Innov 2018; 25:450-4.

14. Horne AW, Phillips JA, Kane N, Lourenco PC, McDonald SE, Williams AR, et al. Cb1 expression is attenuated in fallopian tube and decidua of women with ectopic pregnancy. Plos One 2008; 3:e3969.

15. Downing SJ, Maguiness SD, Tay JI, Watson A, Leese HJ. Effect of platelet-activating factor on the electrophysiology of the human fallopian tube: Early mediation of embryo-maternal dialogue? Reproduction 2002; 124:523-9.

16. Zullo F, Palomba S, Zupi E, Russo T, Morelli M, Cappiello F, et al. Effectiveness of presacral neurectomy in women with severe dysmenorrhea caused by endometriosis who were treated with laparoscopic conservative surgery: A 1-year prospective randomized double-blind controlled trial. Am J Obstet Gynecol 2003; 189:5-10.

17. Donatti L, Ramos DG, Andres MDP, Passman LJ, Podgaec S. Patients with endometriosis using positive coping strategies have less depression, stress and pelvic pain. Einstein (Sao Paulo) 2017; 15:65-70.

18. Cosson M, Querleu D, Donnez J, Madelenat P, Konincks P, Audebert A, et al. Dienogest is as effective as triptorelin in the treatment of endometriosis after laparoscopic surgery: Results of a prospective, multicenter, randomized study. Fertil Steril 2002; 77:684-92.

19. Angioni S, Pontis A, Dessole M, Surico D, De CNC, Melis I. Pain control and quality of life after laparoscopic en-block resection of deep infiltrating endometriosis (die) vs. incomplete surgical treatment with or without $\mathrm{GnRH}-\alpha$ administration after surgery. Arch Gynecol Obstet 2015; 291:363-70.

20. Kim NY, Ryoo U, Lee DY, Kim MJ, Yoon BK, Choi D. The efficacy and tolerability of short-term low-dose estrogen-only add-back therapy during postoperative $\mathrm{GnRH}$ agonist treatment for endometriosis. Eur J Obstet Gynecol Reprod Biol 2011; 154:85-9.

21. Robabeh TMD, Mohammad AKMD, Mohammad GMD. Efficacy of low dose, long-acting gonadotropin releasing hormone analogues (GNRH-a) compared with daily injections of shortacting GNRH-a in ART cycles. Iran J Reprod Med 2004; 2:29-33.

22. Fatima $P$, Hossain MM, Rahman D, Suman GM. Outcome of pregnancies after inadvertent exposure to $\mathrm{GnRH}$ agonist in early pregnancy. Mymensingh Med J 2011; 20:303-7.

23. Roche M, Ibarrola M, Lamberto N, Larrañaga C, García MA. Spontaneous hemoperitoneum in a twin pregnancy complicated by endometriosis. J Matern Fetal Neonatal Med 2008; 21:924-6.

24. Deura I, Harada T, Taniguchi F, Iwabe T, Izawa M, Terakawa N. Reduction of estrogen production by interleukin- 6 in a human granulosa tumor cell line may have implications for endometriosis-associated infertility. Fertil Steril 2005; 83:1086-92.

25. Lee TH, Wu MY, Chen HF, Chen SU, Ho HN, Yang YS. Association of a single dose of gonadotropin-releasing hormone antagonist with nitric oxide and embryo quality in in vitro fertilization cycles. Fertil Steril 2006; 86:1020-2. 